\title{
Research activities and progress on the long pulse ECRH launcher for EAST
}

\author{
Xiaojie Wang ${ }^{1, *}$, Fukun $\mathrm{Liu}^{1}$, Wei $\mathrm{Wei}^{2}$, Yunying Tang ${ }^{1}$, Bo $\mathrm{Li}^{1}$, Liyuan $\mathrm{Zhang}^{1}$, Handong $\mathrm{Xu}^{1}$, Dajun $\mathrm{Wu}^{1}$, Jiafang Shan ${ }^{1}$, \\ Huaichuan $\mathrm{Hu}^{1}$, Jian Wang ${ }^{1}$, Zege $\mathrm{Wu}^{1}$, Wendong $\mathrm{Ma}^{1}$, Jian Zhang ${ }^{1}$, Miaohui $\mathrm{Li}^{1}$, Yang Zhang ${ }^{1}$, Yong Liu ${ }^{1}$ and EAST \\ team
}

${ }^{1}$ Institute of Plasma Physics Chinese Academy of Sciences, No.350 Shushan lake Road, 230031, Hefei, China

${ }^{2}$ Hefei University of Technology, No.193 Tunxi Road, 230009, Hefei, China

\begin{abstract}
A long pulse Electron Cyclotron Resonance Heating (ECRH) system is developed on EAST tokamak for plasma heating and current profile tailoring. The ECRH system is designed to operate at $140 \mathrm{GHz}$ and to inject $4 \mathrm{MW} \mathrm{CW}$ power. With respect to the physical objectives of the newly built ECRH system, a quasi-optical launcher is designed to inject 4MW continuous wave into plasma through an equatorial port. Gaussian beams delivered from evacuated corrugation waveguides will be focused and reflected by high thermal conductive metal mirrors, and then steered by using push-rod steering mechanism with entire scanning range of $\pm 25^{\circ}$ toroidally and over $30^{\circ}$ poloidally in plasma cross section. The mirrors are carefully designed with mega watts power handling capability and optimum optical characteristics. The performance of steering mechanism has been tested before installation, an open-loop control system for ECRH launcher has been implemented for required mirror movement and proper polarization between plasma discharges. This paper will present the overall design and progress of the launcher, along with the performance in EAST campaigns. Considerations and possible upgrade of the design features relevant to long pulse operation are discussed.
\end{abstract}

\section{Introduction}

Electron Cyclotron Resonance Heating (ECRH) scheme has many advantages in magnetic fusion devices, e.g. perfect power coupling with the plasma, highly localized power deposition [1-4] and good theoretical understanding of the wave-particle interaction physics. Besides, due to the technological advantage of launching structure, ECRH will support steady state operation for next step devices, such as ITER [5] and DEMO [6].

As one of the most promising external auxiliary heating and current drive methods, ECRH provides large flexibility and complies with diverse physics demands. It was developed and studied in most tokamaks such as DIII-D [7], ASDEX [8], HL-2A [9] and the stellarators as well, like W7-X [10] and LHD [11]. A long pulse ECRH program is launched on EAST under the support of National Magnetic Confinement Fusion Science Programs of China since 2011. It will be used for central heating and current drive applications as well as off-axis control of magnetohydrodynamic (MHD) instabilities such as Neoclassical Tearing Modes (NTMs), sawtooth, and Edge Localized Mode (ELM). The EAST ECRH system is planning to deliver a total installed power of 4 MW into plasma for either in stationary operation mode with a pulse length up to $1000 \mathrm{sec}$ or a modulation mode with a frequency up to $1 \mathrm{kHz}[12,13]$. Considering the operational range of toroidal magnetic field on EAST and the frequency of a commercially available gyrotron, the new system uses $140 \mathrm{GHz}$ to match the second harmonic of the electron cyclotron resonant frequency.

The ECH power generated from 4 gyrotrons will be transmitted by 4 individual evacuated waveguides and fed to plasma by a front steering quasi-optical launcher from the low field side (LFS) via an allocated equatorial port (M-p) (as shown in Fig.1). Therefore, the launcher should be compatible with the requirements of long pulse power handling and flexible control of localized power deposition. These design goals present a number of challenges. This paper describes the overall design and progress of the launcher.

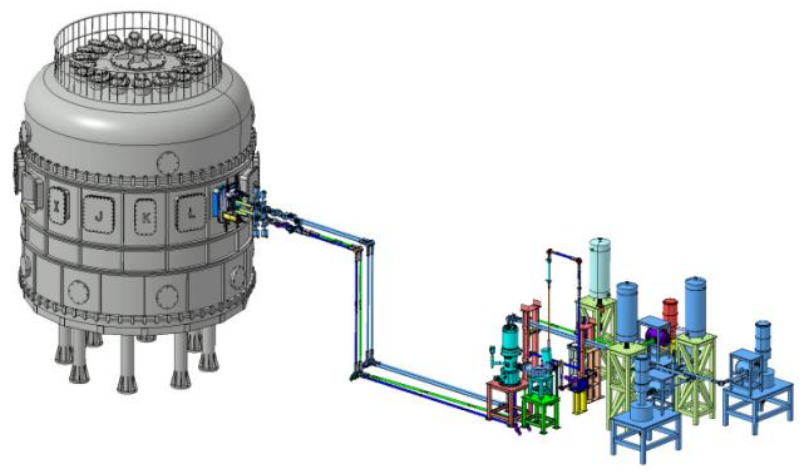

Fig. 1. Schematic of $140 \mathrm{GHz} / 4 \mathrm{MW}$ long pulse ECRH system on EAST

Corresponding author: xjiew@ipp.ac.cn 


\section{Design of launcher}

\subsection{Layout design}

To investigate the operational range of the ECRH system on EAST, numerical simulations of ECCD have been carried out by using the ray tracing code TORAY. For the given equilibrium parameters of EAST, the deposition of EC power was greatly dependent on the scanning of launcher position, and both the poloidal and toroidal steering angles. It is found that a launch from $\mathrm{Z}$ $=30 \mathrm{~cm}$ (the vertical position) is the best scheme for core localized heating and the suppression of neoclassical tearing modes $[14,15]$.

Taken into account the available port dimensions (518 $\mathrm{mm}$ in width and $960 \mathrm{~mm}$ in height), the beam launch position is chosen to locate at $30 \mathrm{~cm}$ vertically away from the equatorial plane and at $\mathrm{R}=3 \mathrm{~m}$ with radial injection. The driven current density and its radial deposition location contours, as a function of the launch angles, are presented in figure 2 . The range of toroidal injection angle from $-25^{\circ}$ to $+25 \circ$ is suitable to obtain higher ECCD efficiency in co-direction or ctr- direction, meanwhile, the beam spans $30^{\circ}$ poloidally, a wide range $(0.03 \mathrm{a} \sim 0.8 \mathrm{a})$ of plasma areas of interest is covered.

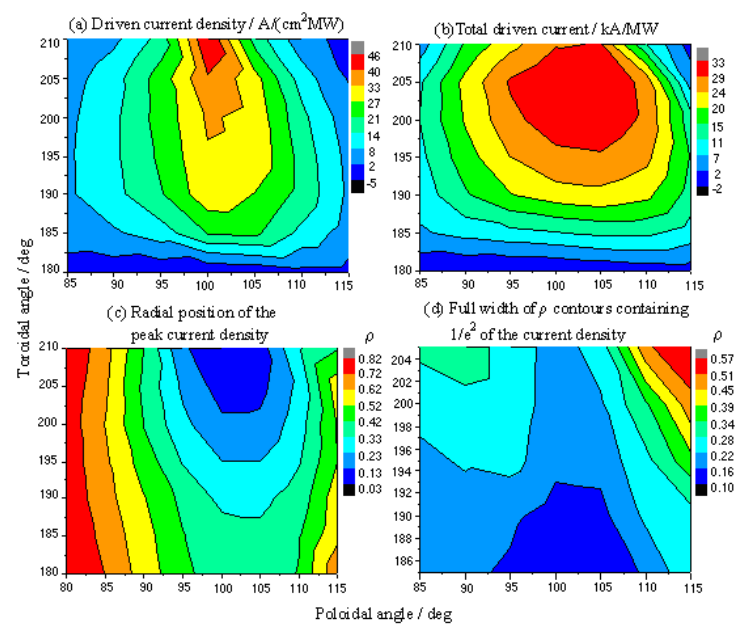

Fig. 2. Two-dimensional variation of ECCD characteristics with different poloidal and toroidal angles when $\mathrm{B}_{0}=2.2 \mathrm{~T}$, Ra $=300 \mathrm{~cm}$, and $\mathrm{Za}=30 \mathrm{~cm}$. (a) Driven current density in units of $\mathrm{A} \cdot \mathrm{cm}^{-2} \cdot \mathrm{MW}^{-1}$, (b) total driven current in units of $\mathrm{kA} \cdot \mathrm{MW}^{-1}$, (c) radial position of the peak current density, and (d) full width of $\rho$ contours containing $1 / \mathrm{e}^{2}$ of the current density

According to the above ray tracing simulation, the launching points of 4 Gaussian beams are symmetrically distributed in two rows and two columns relative to the equatorial plane. The sketch of ECRH launcher is shown in figure 3. Four identical quasi-optical modules are designed for the beams propagating, each contains a 63.5 $\mathrm{mm}$-i.d stainless steel (SSL) corrugated waveguide with interiors nickel plated, a set of quasi-optical arrangement formed by a fixed-focusing and a rotatable-flat mirror. Each beam emitted from the SSL waveguides is obliquely incident on a focusing mirror, and then guided into the flat steerable mirror, which purely changes the beam angle in the poloidal and toroidal directions. All the subassemblies will be integrated to the main frame, an external shield structure is mounted for protection against contamination of lithium (Li) wall coatings.

\subsection{Mirrors design}

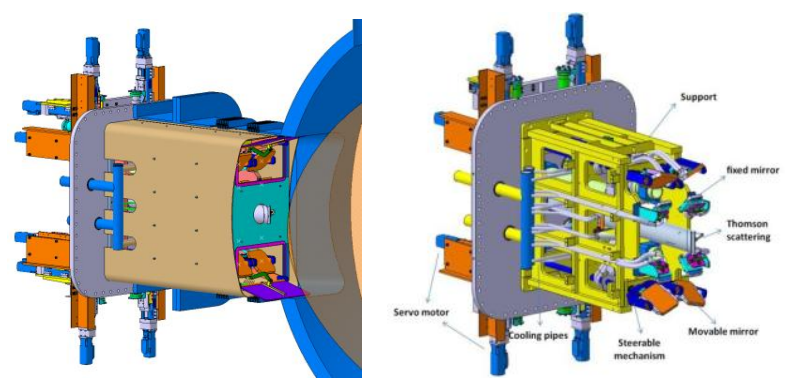

Fig. 3. (Left) Illustration of the $4 \mathrm{MW}$ front steering equatorial launcher design, (Right) Configuration of the launcher

The focusing mirror, at angle of $45^{\circ}$ with respect to the incident beam, allows the possibility of changing the beam size for the higher localized current drive. Spherical focusing mirror was chosen for simplicity, the focal length of the fixed mirror has been optimized in a reference absorption layer in the plasma. The evolutions of the beam waist by varying the focal length under given distance from the SSL waveguide termination to focusing mirror are displayed in figure 4 , The radius of curvature of the spherical mirror is optimized to be 1400 $\mathrm{mm}$, the focusing mirror is located at $240 \mathrm{~mm}$ away from the waveguide output and the beam radius is about 31 $\mathrm{mm}$ at the resonance layer (about $1200 \mathrm{~mm}$ ). Considering the optimized results of the beam propagation properties and the spatial limitation of the port duct, the dimensions of the focusing mirror are $127 \mathrm{~mm} \times 90 \mathrm{~mm}$ and mobile mirror sizes are $170 \mathrm{~mm} \times 113 \mathrm{~mm}$, which are chosen to ensure that at least $99.99 \%$ Gaussian beam power intercepted by reflecting surfaces of mirrors.
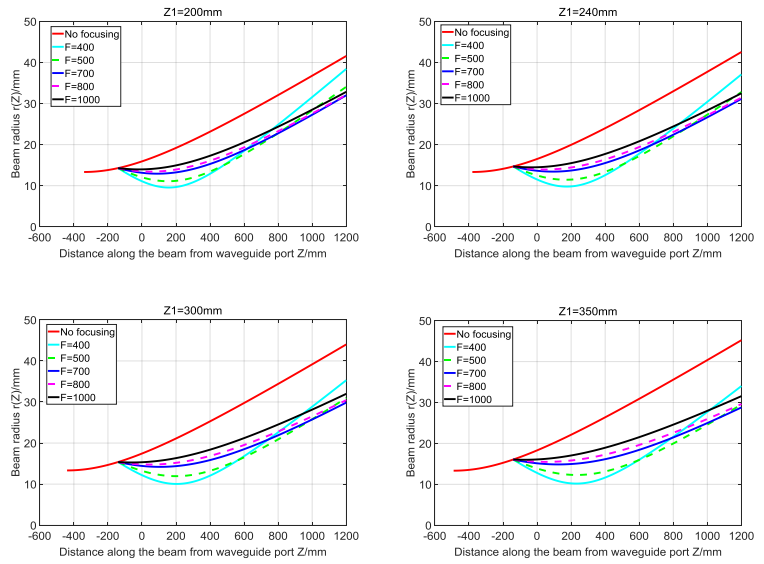

Fig. 4. The beam waist along the propagation trajectory, as a function of shaping mirror focal length. The horizontal -140 $\mathrm{mm}$ and $0 \mathrm{~mm}$ refer to the positions of the focusing mirror and steering mirror, respectively.

The mirrors should be actively cooled to withstand the heat loads during $1 \mathrm{MW}$ long pulse operation. In the initial design, all possible radiation power of EAST future full power operation are estimated for the calculation, therefore, there is big margin of the total heat load. $3 \mathrm{D}$ models of mirrors are set for thermal stress analysis, the choices of material and structure of 
mirrors were guided by thermal calculations and estimations of heat loads on the reflective surfaces [16]. The oxygen-free copper (OFC) is adopted as mirror surface and body for requiring higher thermal conductivity, the parameters of internal cooling channels are carefully optimized to provide sufficient heat exchange with the smooth surface. The heat load on the movable mirror (as shown in figure 5) is higher than on the fixed focus mirror, the cooling channels are cut directly into the copper layer and form several S-shaped loops going from the center to the edge of the mirror. A sheath of stainless steel is backside brazed for higher mechanical robustness. Finite element thermal calculations predict that a maximum temperature is about $296^{\circ} \mathrm{C}$ located at the center of the mirror surface and the peak stress is $178.1 \mathrm{MPa}$ at the corner of cooling channels, the peak temperature and stress are allowable.

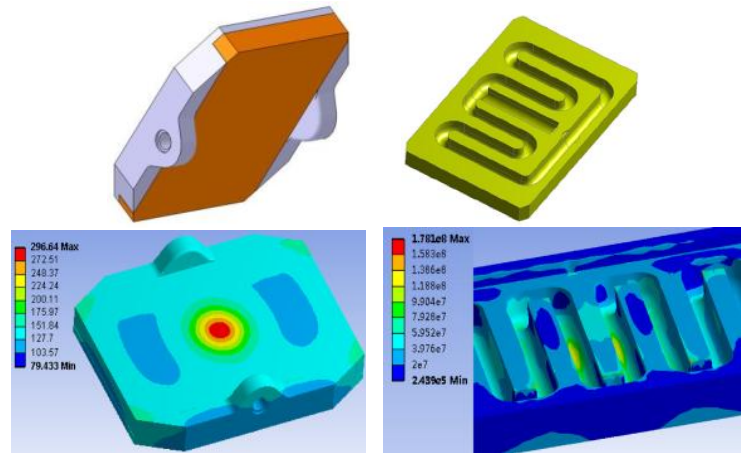

Fig. 5. (Upper) The cooling design of the mobile mirror, (Lower) the temperature and the stress distributions of the mirror.

The uniform thickness is $20 \mathrm{~mm}$ for the flat mobile mirror, the choice of the metal material and increased mass are often accompanied by high electromagnetic forces. The distribution of magnetic field on the mirror, induced current density, force and torque are computed by MAXWELL software and the structure stress and deformation of mirror are analyzed in the case of plasma current quench with exponential decay [17]. The result of the electromagnetic transient analysis indicates that the mirror could work safely under the strong electromagnetic environment. A maximum deformation as seen from figure 6 , locates on the top of mirror is $0.0225 \mathrm{~mm}$ which is very small with respect to the size of mirror. However, to decrease the induced eddy current density, force and torque on the edge of the mirror, design modification of mirrors is under investigating, such as further optimizing the cooling design to decrease the heat volume with high conduction, trying a tapered thickness mirror to reduce electromagnetic force.
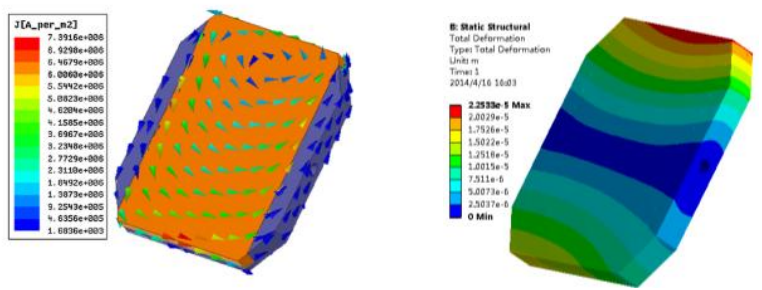

Fig. 6. The distribution of induced eddy current density and the maximum deformation locates on the top of mirror

\subsection{Steering mechanism and performance}

A pair of steering mechanisms for the mobile mirror enables extensive control of the toroidal and poloidal injection angles for the beam. As shown in figure 7, the two directional steering motions are respectively actuated by horizontal motor and vertical motor, the angle steering is achieved by a push-rod drive [18]. The poloidal injection degree could be changed independently by pushing or retracting the rod, no coupling happens during poloidal movement when toroidal is fixed. The toroidal movement is realized through the rotation of rod around its axis, the connecting rod outside the vessel transmits the up-anddown motion to the crankshaft, which changes it into rotary motion inside the vacuum box. A slight poloidal motion will occur when scanning the mirror toroidally, the poloidal angle has to be corrected accordingly. All rods are connected via welded bellow feed-throughs to servo-motors situated outside the port flange.

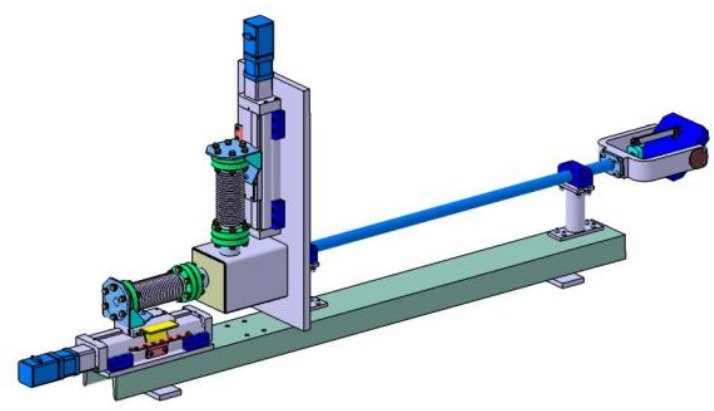

Fig. 7. 3D view of the steering mechanism design

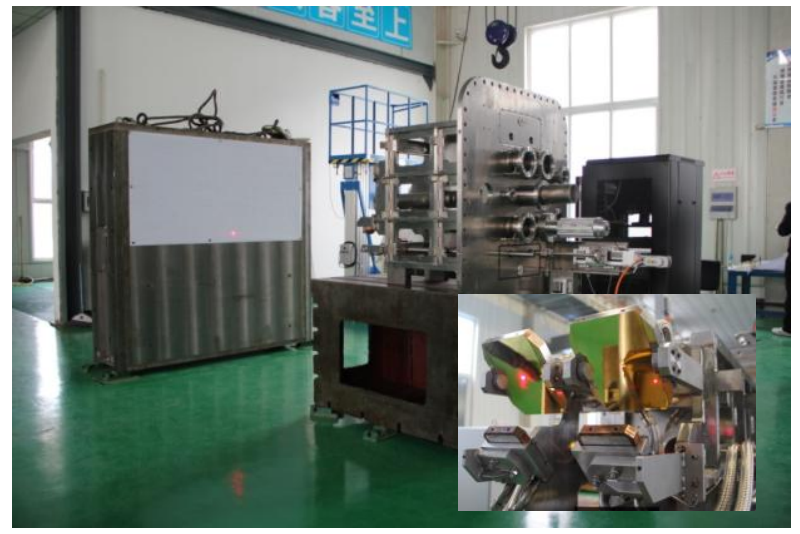

Fig. 8. Optical calibration of the launcher

The two directional steering angles are accomplished via independent actuator rods. To determine the transformation functions of beam angles and displacements of pushrods, the configuration of the space linkage driving mechanism has been mathematically analyzed by using the direction of the normal to the steerable mirror and the geometry of reflection [19]. The sophisticated mathematical relationships between the displacements of linear driving sliders on both directions and the corresponding shift angles of reflected beam was derived, the kinematic analysis was programmed by Matlab and integrated in the launcher programmable logic controller (PLC) 
control system. Laser calibrations have been implemented to test the steering performances and the alignment of the components (as shown in figure 8).

The graph in figure 9 displays the scanning map and the test results on the front projective plane. The sector diagram is obtained based on the correlation analysis of steering structure, in which the longitude lines represent the calculated trajectories of poloidal angles with several given toroidal angles, while the latitude lines denote the toroidal scanning. During the test, the theoretical positions of the randomly selected injection angles were marked with stars. The positions of laser points were marked with triangular symbols, the purple one and the light blue one represent the back and forth moving directions, respectively. It is obvious that the tracking routes of laser points are in good agreement with the dotted theoretical lines, hence, the steering feasibility and validity of the expressions were confirmed, it can provide a reliable mathematical model for the accurate control of beam steering. Whereas, the deviation seems increasing when the mirror oriented at its steepest angle relative to the incident beam, it is particularly sensitive to the toroidal angle. The maximum deflection of the angle is about $0.5 \mathrm{deg}$. One of the factors which affect the accuracy is that the fit clearance and the elastic deformation of the slender shaft were ignored in the mechanical kinematic analysis, however, the exiting tolerance of all the machining parts certainly led to the positioning bias. The torque caused by the plastic deformation of the flexible metal cooling pipe also gives rise to the backlash of the rotary motion. Moreover, the beam is assumed to ideally inject on the center of the mirrors, a small displacement of the mirror arrangement will consequentially result in undesired shift of the beam deposition on the target area.

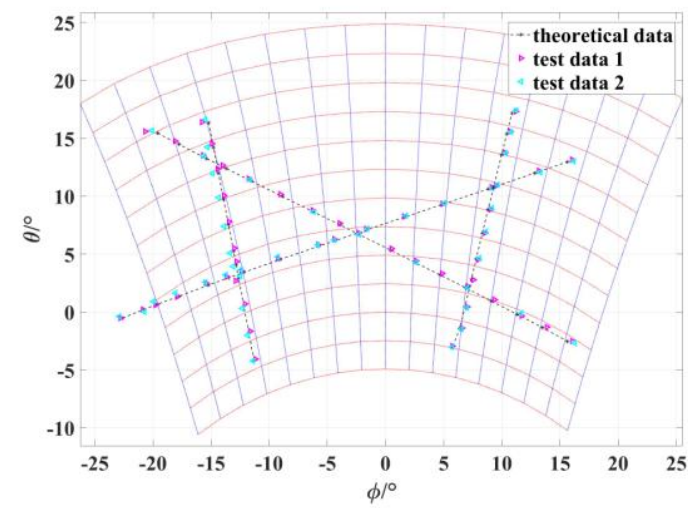

Fig. 9. Scanning map of EAST ECRH launcher on the projective target (distance $\mathrm{L}$ from the launching point is $2 \mathrm{~m}$ )

To procure a launcher that fulfills the requirements of EAST in terms of performance and system reliability, Tests activities including the steering optics, actively cooling capability, the thermomechanical and fatigue aspects of the design should be carried out during the R\&D phase for design validation prior to installation in EAST port. In particular, the vacuum seal performance of launcher with pressurized cooling pipes was rigorously inspected in a testing chaember under vacuum and $200^{\circ} \mathrm{C}$ baking conditions for the sake of security operation.

Errors in the positioning of the mirrors and the deformations of the port plug structure will also influence the launch points and injection angles, therefore, validation test of optics after installation was performed to inspect the impact of possible misallignment.

\subsection{Shutter design}

In EAST campaigns, lithium (Li) powder evaporation was used as a routine wall conditioning technique to access the H-mode during plasma discharges. In order to protect the mirrors surface and steering mechanism against coating contamination by deposited Li powder, a tight mandatory metal shield is designed. The critical part of the shield structure is the movable shutter in front of the beam propagating route. Figure 10 shows the schematic drawing of the shutter, two pieces of SSL plates are used and pneumatic actuated by cylinder, a long driveshaft with a crank links to the plate, the linear motion of the rod generates the plate rotary along its fixed edge. Considering the required angular range of plate motion, the travel distance of the rear slider was carefully designed and positioned. The movable performance was tested using a prototype under the bulk lithum coating experiment during $R \& D$, the shutter can be smoothly open and close after lithum coating.

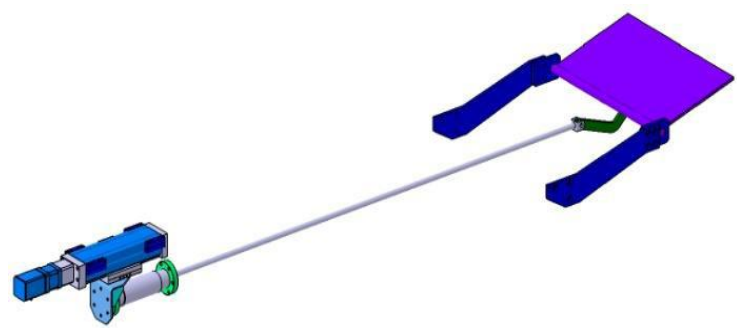

Fig.10. Schematic drawing of the shutter

\section{Progress of launcher}

\subsection{Current status}

The fabrication of the launcher was in two steps, the lower two channels of the launcher were developed and installed on EAST in 2014 without power. The lower right module of the launcher for power injection has been in service since 2015 spring campaign till now. The launcher with all 4 modules was built in last year, it is presently installed on EAST as shown in figure 11. In the coming experiment, the second gyrotron from GYCOM is tested and ready for operation, another launcher module will be employed and await its initial operation. 

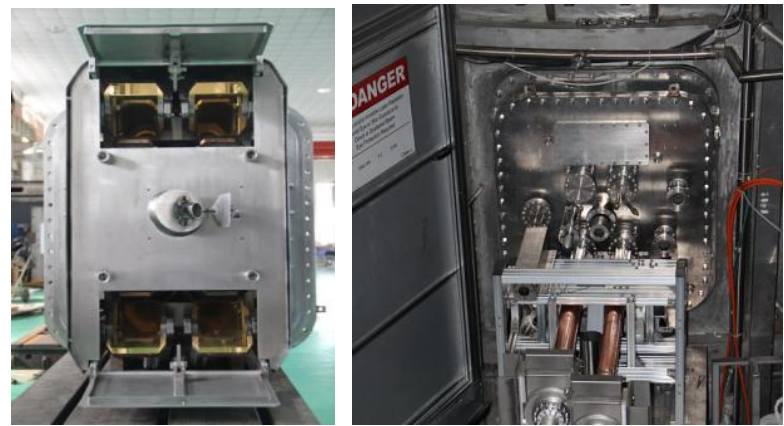

Fig.11. Photos of the launcher plug-in

\subsection{Engineering commissioning}

The commissioning of launcher in situ was done during the initial operation of $1 \mathrm{MW}$ ECRH system powered by \#1 gyrotron. Figure 12 indicates the experimental result of power scanning capability of the first launcher under a modulated operation at frequency of $10 \mathrm{~Hz} / 1 \mathrm{~s}$. Nine sets of beam angles varied in a sequence of discharge shots, the deposit locations were measured by ECE diagnostics. The obtained scanning range is accessible from core to boundary close to 0.8 . During the test, no obvious hot spots were found in the high filed wall which confirmed that polarizations and injection angles were as expected [20]. Comparing the measured deposition positions with the predicted value, most of them agree well except some offset around 0.3 , the experiment will be further done to identify the position precision. Till now, the launcher is stably operated under $100 \mathrm{~s}$ pulse length with $500 \mathrm{~kW}$ injection power. The influence on sawtooth period and tearing mode stabilizing were investigated due to the flexible steering capability [21].
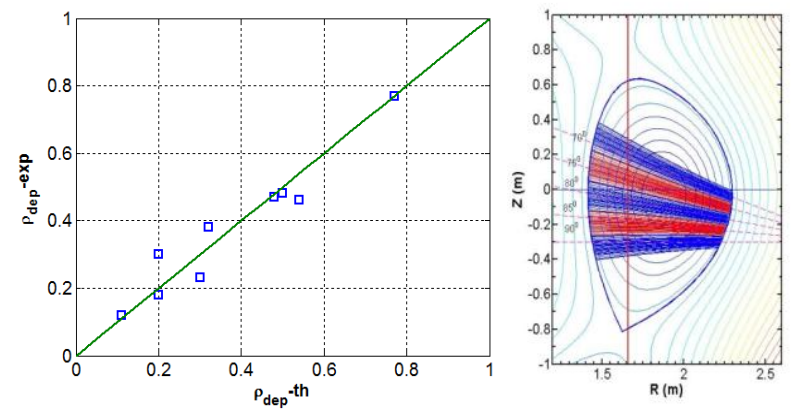

Fig.12. Measured Versus Calculated Deposition Radius by $10 \mathrm{~Hz} / 1 \mathrm{~s}$ modulated ECRH

\subsection{Performances improvement}

\subsubsection{Launcher control}

The control of the power deposition is anticipated to feedback control of the steerable mirrors. In the steering calibration test, the velocity and the response time of the steering mechanism are primarily tested, the obtained velocity is about $5 \% / 50 \mathrm{~ms}$ in poloidal. The direct current servomotor has an adequate ratio of torque to inertia, but for the purpose of fast scanning, more work should be done to optimize the mechanical structure to reduce the friction of the pivot joints. The divation result from the mechanical tolorenance and backlash needs to be investigated and appropriately compensated in the algorithm. In addition, the mirror mounting structures and the dynamic tracks of cooling bellows will be further optimized to facilitate the position adjustment during the optical calibration, and minimize the slight angular misalignment. Hence, the position accuracy and the steering velocity need to be further determined.

The launcher steering is remote controled by a control loop based on PLC system. At present, the beam deposition is feed forward controlled, the mirror angles are adjusted shot by shot during discharge experiment. The PLC system works for mirror movement control as well as monitoring the general status of the motors. The aiming process was not fully automated, yet, a database for the objective ECCD deposition position is off-line computed by TORAY. The target depositions could be achieved by manually setting injection angles on the control screen, the steering commands are dispatched through the PLC to the steering mirror drives, the encoders transform them into the motion parameters and trigger the motor to implement it.

Fast poloidal steering is important for rapid tracking of instabilities. However, at the moment, the response time of polarization control is nearly $1 \mathrm{sec}$, which is not fast enough for real time operation. In order to meet the requirement of NTM suppression, a fast poloidal scan within small range $\left( \pm 4^{\circ}\right.$ around the initial position of mirror) was developed to sweep the ECCD in a small step for a better alignment to the island with no need of polorization change, the angular resolution of the mirror rotation is desired to be better than $1^{\circ}$. The representative fast scan was tested in 2017 EAST campaign, the achieved angular velocity obtained from the response in plasma is $2^{\circ} / 200 \mathrm{~ms}$.

In the near term plan, a mock-up shown in figure 7 will be fabricated and actuated with a control modular (in figure 13), extensive test will be excuted for further analysis and optimization of the steering performance. Improvement of the control system by integrating real time calculations of RF deposition using fast real time EFITs and kinetic profiles using an accurate diffraction model will be part of future work.

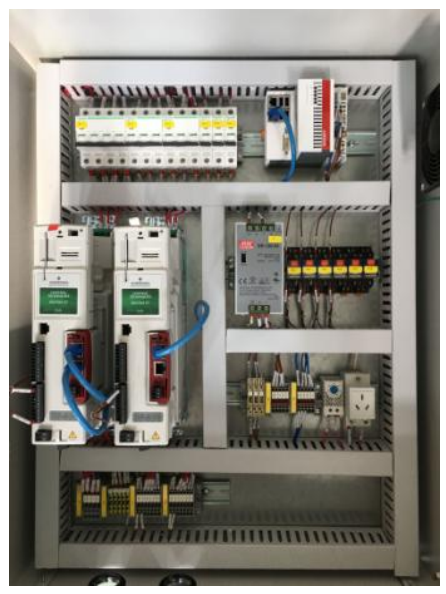

Fig. 13. Control modular of the steering mirror 


\subsubsection{Mirror replacement}

After each campaign with lithum wall conditionning, the deposition of dust or lithium could be found on the surface of the mirrors, especially the steering mirror (as shown in figure 14), which are need to be replaced or cleaned. To remove the mirror, the flexible cooling tube connected with the mirror should be cut off, and the steering mechanism should be taken apart. After new mirror mounted, the mechanism and the cooling should be re-assembled, the leakage test as well as the optical and mechanical test should be repeated to ensure the reliability of the launcher. The tricky issue encountered in the burdensome maintenance process is the copper mirror surface protection. Now, a novel design concept of detachable mirror surface is ongoing to simplify the mirror replacement work.
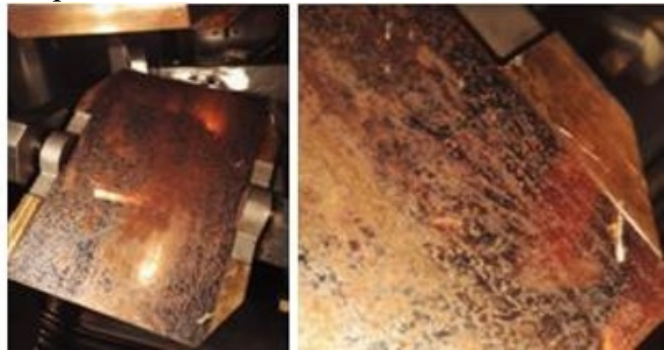

Fig. 14. Contaminations on the steering mirror surface

\section{Summary}

A long pulse quasi-optical launcher rated for $4 \mathrm{MW} /$ $1000 \mathrm{sec}$ power injection has been developed on EAST ECRH system, the design is overviewed in this paper. Efforts have been made on the design of the most critical elements such as steering mechanism of the mirror and shutter, flexible cooling connection for mobile mirror, a series steering mechanism test and optical calibration of the rotation angle. Gaussian beams delivered from evacuated corrugation waveguides will be focused and reflected into designated plasma area by actively cooled metal mirrors, with entire scanning range of $\pm 25^{\circ}$ toroidally and over $30^{\circ}$ poloidally. The mirrors are carefully designed to satisfy the desired physical requirements. Characteristics of beam zooming and propagation has been optimized, the thermal-mechanical evaluation has been performed by finite element analysis software. Test activities including beam steering, cooling and leakage inspection have been done before installation. Based on the commissioning results, Attentions are paid on steering performance improvement and development of the open-loop control system towards real time feedback control ability.

\section{Acknowledgement}

This work supported by the National Magnetic Confinement Nuclear Fusion Power Development Special Fund of China (Grant Nos. 2011GB102000, 2012GB103000 and 2015GB102004). The authors greatly appreciate all kind person from the following laboratories: GA, PPPL, Tore Supra and NIFS for the fruitful discussions.

\section{References}

1. H. Zohm, Fusion Sci. Technol. 52 (2007) 134-144.

2. M. Bornatici, et al., Nucl. Fusion 23 (1983) 1159

3. V. Erckmann, Plasma Phys. Controlled Fusion 36 (1994) 1869

4. R. Prater, Phys. Plasmas 11 (2004) 2349.

5. T. Omoria, Fusion Eng. Des. 86 (2011) 951-954

6. E. Poli, Nucl. Fusion 53 (2013) 013011

7. J. Lohr, J Infrared Milli Terahz Waves 32 (2011) 253-273

8. J. Stober, EPJ Web of Conferences 201232020 (2012)

9. X. R. Duan, Nucl. Fusion 50 (2010) 095011

10. V. Erckmann, et al., Fusion Sci. Technol. 52 (2007) 291-312

11. Yoshimura, et al., Fusion Sci. Technol. 58 (2010) 551

12. X. J. Wang, et al., Fusion Eng. Des. 96-97 (2015)

13. H. D. Xu, et al., Plasma Sci. Technol. 18 (2016) 442

14. W. Wei, et al., Chin. Phys. B 23 (2014) 055201

15. X.G. Wang, et al., Phys. Scr. 86 (2012) 065501

16. Y. Tang, et al., Fusion Eng. Des. 89 (2014) 847

17. Y. Tang, et al., J Fusion Energy 34 (2014) 422-426

18. R Ellis, et al., Fusion Engineering 1-4 (2011)

19. L. Zhang, et al., Machine Des. Research 31 (2015) 5

20. D. J. Wu, et al., J Fusion Energy 33 (2014) 634-639

21. Y. Yuan, et al., Phys. Plasmas 23 (2016) 062503 